\title{
SLC25A22 promotes proliferation and metastasis by activating MAPK/ERK pathway in gallbladder cancer
}

\author{
Pengcheng $\mathrm{Du}^{1,2+}{ }^{-0}$, Haibin Liang ${ }^{3 \dagger}$, Xiaowei Fu ${ }^{4 \dagger}$, Peng Wu${ }^{1}$, Chao Wang ${ }^{1}$, Haimin Chen ${ }^{1}$, Bingbing Zheng ${ }^{1}$,
} Jun Zhang ${ }^{1}$, Shuanghui Hu' ${ }^{1}$, Rengui Zeng ${ }^{1}$, Bo Liang ${ }^{1}$ and Lu Fang ${ }^{1,2^{*}}$

\begin{abstract}
Background: SLC25A22, a member of mitochondrial carrier system (MCS) family encoding a mitochondrial glutamate transporter, has been reported to have vital roles in promoting proliferation and migration in cancer. Gallbladder cancer (GBC) is the most common biliary tract malignancy and has a poor prognosis. We aimed to determine the expression and function of SLC25A22 in GBC.

Methods: Immunohistochemistry $(\mathrm{IHC})$ staining analysis and quantitative real-time PCR (qRT-PCR) were conducted to determine the expression of SLC25A22 in GBC tissues. Human NOZ and GBC-SD cells were used to perform the experiments. The protein expression was detected by western-blot analysis. Cell viability was evaluated via CCK-8 assay and colony formation assay. Cell migration and invasion in vitro were investigated by wound healing and transwell assay. Annexin V/PI staining assay for apoptosis were measured by flow cytometry. The effect of SLC25A22 in vivo was conducted with subcutaneous xenograft.
\end{abstract}

Results: We indicated that the expression of SLC25A22 was significantly upregulated in GBC tumor tissues as well as cell lines. Downregulation of SLC25A22 inhibited GBC cell growth and proliferation in vitro and in vivo and also had an effect on metastasis of GBC cells through the EMT processes. In addition, inhibition of SLC25A22 promoted mitochondrial apoptosis via downregulating BCL-2 and upregulating cleaved PARP, Cytochrome-C, and BAX mediated by MAPK ERK pathway.

Conclusions: Our study identified that SLC25A22 promoted development of GBC activating MAPK/ERK pathway. SLC25A22 has a potential to be used as a target for cancer diagnosis of GBC and related therapies.

Keywords: Gallbladder cancer, SLC25A22, Mitochondrial apoptosis, MAPK/ERK

\section{Background}

Gallbladder cancer (GBC) is the most common biliary tract malignancy and the fifth most common gastrointestinal cancer [1]. The poor prognosis of GBC is due to its usually delayed diagnosis and early metastasis, as the 5 -year survival rate is only $\sim 5 \%[2,3]$. Therefore, there a limited time period in which a patient can receive

\footnotetext{
*Correspondence: fanglu@medmail.com.cn

${ }^{\dagger}$ Pengcheng Du, Haibin Liang and Xiaowei Fu contributed equally to this work

${ }^{1}$ Department of General Surgery, Second Affiliated Hospital of Nanchang University, No. 1 Minde Road, Nanchang 330006, China

Full list of author information is available at the end of the article
}

surgical treatment. As a result, a better understanding of the molecular mechanism of GBC is indispensable.

The 53-member canonical SLC25A transporter groups and several identified noncanonical transporters are involved in the mitochondrial carrier system (MCS) in mammals. This system transports small molecules between the mitochondria and cytoplasm [4]. SLC25A22 specifically, as a member of MCS family, was identified to encoded a mitochondrial glutamate transporter [5]. Recent studies have reported on the potential effects of SLC25A22 in neonatal epileptic encephalopathy and migrating partial seizures in infancy [6-9]. In addition, Wong and $\mathrm{Li}$ et al. revealed that the overexpression of

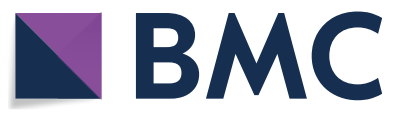

(c) The Author(s) 2019. This article is distributed under the terms of the Creative Commons Attribution 4.0 International License (http://creativecommons.org/licenses/by/4.0/), which permits unrestricted use, distribution, and reproduction in any medium, provided you give appropriate credit to the original author(s) and the source, provide a link to the Creative Commons license, and indicate if changes were made. The Creative Commons Public Domain Dedication waiver (http://creativecommons.org/ publicdomain/zero/1.0/) applies to the data made available in this article, unless otherwise stated. 
SLC25A22 in colorectal cancer (CRC) and its vital roles in promoting proliferation and migration of CRC with mutation KRAS. Meanwhile, elevated expression of SLC25A22 was associated with a poor prognosis [10]. However, there is little research about SLC25A22 in malignant tumors and no study of effects and mechanism of SLC25A22 in GBC.

In this study, we determined the expression of SLC25A22 in GBC and further explored its function in the GBC cell. Moreover, we suggested that MAPK/ERK pathway was affected by SLC25A22 thereby mediating mitochondrial apoptosis to promote malignant behaviors in GBC. Taking together, SLC25A22 has potential to be a prognostic and therapeutic biomarker in GBC.

\section{Methods}

\section{Cell culture}

The GBC lines used in this study were as follows: GBCSD, SGC-996 were purchased from Shanghai Institute for Biological Science, Chinese Academy of Science (Shanghai, China). NOZ, OCUG-1, EHGB-1, EHGB-2 were purchased from the Health Science Research Resources Bank (Osaka, Japan). GBC-SD, EHGB-1, EHGB-2, OCUG-1 cells were cultured separately in DMEM (Gibco) with $10 \%$ fetal bovine serum (FBS, Gibco) and 1\% penicillinstreptomycin (Gibco). NOZ cells were cultured in William's medium E (Lonza) with $10 \%$ fetal bovine serum (FBS, Gibco) and 1\% penicillin-streptomycin (Gibco). Contrastingly, SGC-996 cells were cultured in RPMI 1640 medium (Hyclone) with $10 \%$ fetal bovine serum (FBS, Gibco) and 1\% penicillin-streptomycin (Gibco). All of above cells were cultured in their respective media in a humidified incubator at $37^{\circ} \mathrm{C}$ with $5 \% \mathrm{CO}_{2}$.

\section{Immunohistochemistry (IHC)}

Immunohistochemical staining of GBC and paracancerous tissue was performed using anti-SLC25A22 (Abcam, 1:200), and subcutaneous xenograft of mice using antiKi67 (Abcam, 1:200) and anti-p-ERK (CST, 1:200). The sections were incubated with primary antibody overnight at $4{ }^{\circ} \mathrm{C}$ after deparaffinization and antigen recovery. Then, the sections were incubated with a secondary antibody (Beyotime, 1:2000) for about $40 \mathrm{~min}$ at $20{ }^{\circ} \mathrm{C}$. Sections were scored as previously described [11].

\section{Quantitative real-time PCR (qRT-PCR)}

Total RNA was extracted from tissues by using Trizol reagent (Invitrogen), thus, the cDNA products were synthesized using PrimeScript Reverse Transcriptase (Takara) according to the manufacturer's protocol. All products were amplified by PCR and measured using SYBR ${ }^{\circledR}$ Green (Takara), the expression results were analyzed by StepOnePlus ${ }^{\mathrm{TM}}$ Real-time PCR system (Applied
Biosystems). The primer sequences for SLC25A22 were shown as follows: $5^{\prime}$-primer, GGGAGTTCACCGGAC ATCTG and 3'-primer, CCCATTCAATCTCCCGCT GT. The relative expression was estimated with $2^{-\Delta \Delta C T}$ method, using GAPDH as an internal control.

\section{Migration and invasion assays}

Cell migration and invasion were assessed using transwell migration and invasion chambers (BD Biosciences). Cells were transfected with shRNA-SLC25A22 and shRNA$\mathrm{NC}$ were resuspended in serum-free medium and then seeded respectively into the upper chambers, while the lower chambers was maintained in $10 \%$ FBS medium. After $20 \mathrm{~h}$ of incubation, cells on the bottom of chambers were fixed with $4 \%$ paraformaldehyde for $30 \mathrm{~min}$ and dyed with crystal violet for $15 \mathrm{~min}$. The images of stained cells were captured using a microscope (Leica).

\section{Cell viability assay}

Cell viability was assessed using Cell Counting Kit-8 (CCK-8, Yensen) according to the manufacturer's instructions. Firstly, cells were separately seeded into 96-well plates at a density of 1000 per well. After incubation with CCK- 8 for $2 \mathrm{~h}$, the absorbance of cells was measured at a wavelength of $450 \mathrm{~nm}$ by a microplate reader (BioRad), all of above were conducted at various times $(6 \mathrm{~h}, 24 \mathrm{~h}$, $48 \mathrm{~h}, 72 \mathrm{~h}, 96 \mathrm{~h}$ ). The results were demonstrated in the form of a line chart.

\section{Colony formation and wound-healing assay}

Cells were separately plated into six-well plates at a density of 1000 per well with 10 days of incubation to conduct colony formation. Cell formations were fixed with $4 \%$ paraformaldehyde for $30 \mathrm{~min}$, and subsequently stained with crystal violet and the total number of colonies (more than 50 per colony) was counted. For woundhealing assay, cells were seeded into six-well plates and grown to confluence, then wounds were created by scratching confluent cell monolayers with a $200-\mu \mathrm{l}$ pipette tip and incubating them with $10 \%$ FBS medium in incubator at $37^{\circ} \mathrm{C}$ with $5 \% \mathrm{CO}_{2}$. Images were taken at $0 \mathrm{~h}$ and $24 \mathrm{~h}$ under a microscope (Leica). The percentage (\%) change in migration was determined via comparison of the difference in wound width.

\section{Western-blot}

Total protein was extracted from cells using RIPA buffer (Beyotime) at $4{ }^{\circ} \mathrm{C}$ about 20 min while the PMSF (Beyotime) was used for the inhibition of protein degradation. Equal amounts of sample protein (15-20 $\mu \mathrm{g}$ per lane) were separated by SDS-PAGE and transferred onto $0.45 \mu \mathrm{m}$ PVDF membranes (Millipore). After blocked with $5 \%$ milk at $20{ }^{\circ} \mathrm{C}$ about $1 \mathrm{~h}$, membranes were 
incubated with the corresponding primary antibody at $4{ }^{\circ} \mathrm{C}$ overnight, followed by three times wash in TBST for $10 \mathrm{~min}$. After that, the membranes were incubated with HRP-conjugated secondary antibodies (Beyotime) at $20{ }^{\circ} \mathrm{C}$. Protein bands were exposed by the Gel Doc 2000 (BioRad). The primary antibodies mentioned above were for: SLC25A22 (Abcam, 1:1000), E-cadherin (Abways, 1:1000), N-cadherin (Abways, 1:1000), vimentin (CST, 1:1000), ERK (CST, 1:1000), p-ERK (CST, 1:1000), MEK (CST, 1:1000), p-MEK (CST, 1:1000), GAPDH (Abways, 1:1000), BCL-2 (ABclonal, 1:1000), cytochrome-c (CST, 1:1000), PARP (CST, 1:1000), cleaved PARP (CST, 1:1000).

\section{Lentivirus and cell infection}

For the knockdown of SLC25A22, a short hairpin RNA (shRNA-SLC25A22) sequence complementary to SLC25A22 (5'-GTGGTGTACTTCCCGCTCTTT-3') and a scramble sequence were synthesized by Genomeditech. Cells were harvested $48 \mathrm{~h}$ after transfection of lentivirus. When green fluorescence $(>80 \%)$ was observed under fluorescence microscope, which indicated the establishment of stable transfection. The efficiency of this shRNA was examined by western-blot as mentioned previously.

\section{Subcutaneous xenograft}

Fourteen BALB/c nude mice (average 4 weeks-old and $20 \mathrm{~g}$ ) were purchased from the Shanghai Laboratory Animal Center of the Chinese Academy of Sciences (Shanghai, China) and were divided into two groups randomly. $\mathrm{NOZ}$ cells ( $\mathrm{Lv}-\mathrm{NC} / \mathrm{Lv}-\mathrm{SLC} 25 \mathrm{~A} 22)$ were injected into the armpit of each mouse at a density of $1 \times 10^{6} / 100 \mu \mathrm{l}$. Tumor volumes were measured twice a week from what they were visible. About 4 weeks later, all mice were killed and tumor tissues were fixed by $4 \%$ paraformaldehyde for IHC staining analysis. All operations as described above were approved the Institutional Animal Care and Use Committee of Xinhua Hospital, School of Medicine, Shanghai Jiao Tong University.

\section{Cell apoptosis assay}

An Annexin V/PI Apoptosis Kit (BD Biosciences) was used for cellular apoptosis analysis, NOZ cells $(\mathrm{Lv}-\mathrm{NC} /$ Lv-SLC25A22) were harvested and washed with cold phosphate buffer saline (PBS), then the cells were resuspended in $1 \times$ Binding Buffer at a concentration of $1 \times 10^{6}$ cells $/ \mathrm{ml}$ and $100 \mathrm{ul}$ of the solution $\left(1 \times 10^{5}\right.$ cells $)$ was transferred to a $5 \mathrm{ml}$ culture tube. After incubation with $5 \mu \mathrm{l}$ FITC Annexin V and $5 \mu \mathrm{l}$ PI in the dark for $15 \mathrm{~min}$ at $25^{\circ} \mathrm{C}$, the samples were mixed into $400 \mu \mathrm{l}$ $1 \times$ Binding Buffer and immediately analyzed by flow cytometry (BD Biosciences).

\section{Results}

\section{SLC25A22 overexpression be related with poor prognosis} in GBC

Research of Wong et al. showed that SLC25A22 had a critical role in promoting proliferation and migration in colorectal cancer. In the light of our microarray results, we hypothesized that overexpression of SLC25A22 may play a similar role in GBC. To test this conjecture we compared the expression of SLC25A22 in GBC tissues and corresponding adjacent normal tissues via IHC (Fig. 1a). We found that the expression of SLC25A22 in tumor tissues was significantly higher than that in adjacent tissues. In addition, the results of PCR also demonstrated that there was a difference at the RNA level (Fig. 1b).

\section{SLC25A22 expression level in GBC cell lines}

Western-blot assay was used to detect the protein expression level in six gallbladder cancer cell lines (NOZ, GBCSD, EHGB-1, SGC-996, OCUG-1, EHGB-2). As a result, GBC-SD and NOZ were chosen as candidates because they had higher level of SLC25A22 than other cell-types (Fig. 1c). To validate the function of SLC25A22 in GBC cells, we synthesized a lentiviral shRNA specially against SLC25A22 which could effectively suppress the expression of SLC25A22. Knockdown efficiency of SLC25A22 were tested in mRNA and protein level by qRT-PCR and western-blot (Fig. 1d, e).

\section{Knockdown of SLC25A22 inhibited GBC cell growth and proliferation in vitro and in vivo}

In order to reveal the role of SLC25A22 in cell growth and proliferation, we downregulated the expression of SLC25A22 in GBC-SD cells and NOZ cells via transfecting with lentiviral shRNA. Cell viability was tested by CCK-8 assay and colony formation assay was conducted as described previously (Fig. 2). In contrast, downregulation of SLC25A22 obviously inhibited the viability of GBC-SD cells and NOZ cells. Moreover, colony formation assay demonstrated that the number of colony form was also decreased by the block of SLC25A22.

In order to further verify the suppressive effect on tumor growth in vivo via downregulation of SLC25A22, we established a stable NOZ cell line with low expression of SLC25A22 by transfecting lentiviral shRNA while shNC was a control. Then the two groups of NOZ cells (shSLC25A22/shNC) were subcutaneously injected into node mice and the rate of tumor growth were recorded (Fig. 3a, b). As expected, knockdown of SLC25A22 significantly inhibited the subcutaneous xenograft growth. Consistently, IHC staining of the proliferative index Ki67 and p-ERK displayed a similar result (Fig. 3c, d). In 
a

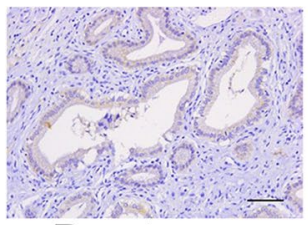

Paratumor

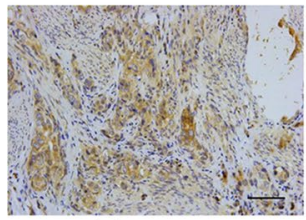

Tumor

b

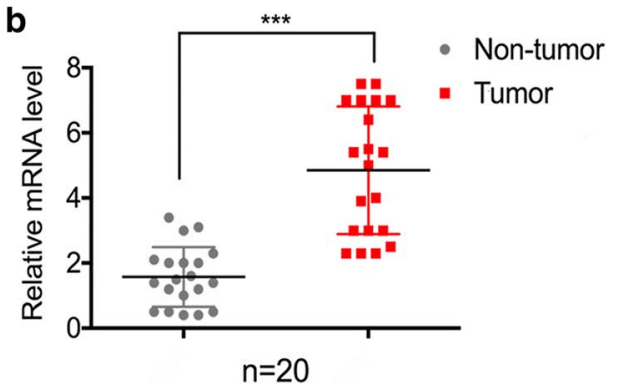

d
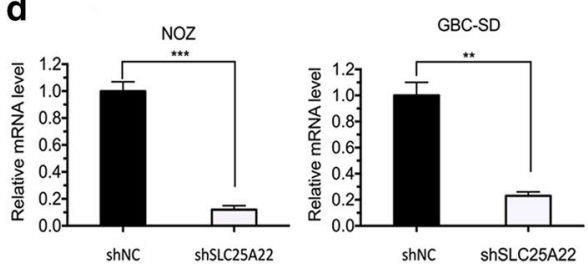

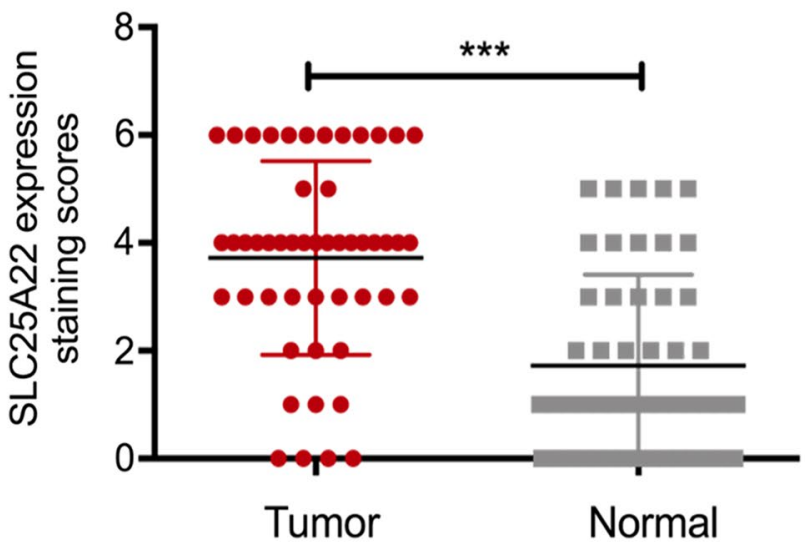

C
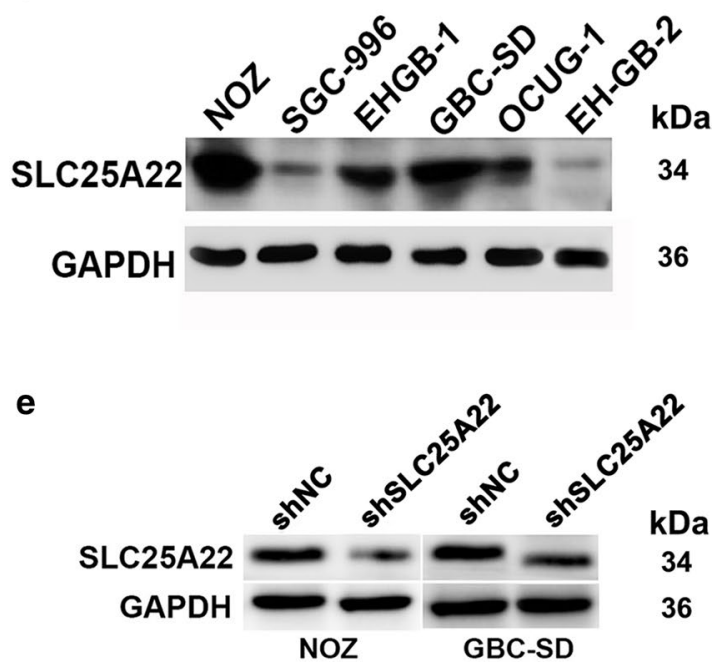

Fig. 1 SLC25A22 is overexpressed in gallbladder cancer (GBC). a Immunohistochemical staining of tumor tissues and corresponding adjacent normal tissues for SLC25A22. Expression staining scores are shown in the graph bar $(n=40)\{p<0.001\}$. b Levels of relative SLC25A22 mRNA were detected in $20 \mathrm{GBC}$ tumor tissues and corresponding normal tissues by qRT-PCR $(n=20)\{p<0.001\}$. c NOZ and GBC-SD display higher expression of SLC25A22 than others, as observed by western-blot. $\mathbf{d}$, e Knockdown efficiency of an shRNA specifically directed against SLC25A22 was tested in protein and mRNA level. An shRNA blocked relative mRNA of SLC25A22 compared to control (shNC) in NOZ and GBC-SD using qRT-PCR, western-blot. The expression of SLC25A22 protein was significantly decreased in NOZ and GBC-SD transfected with shRNA. GAPDH was used an internal reference

summary, SLC25A22 may play a crucial role in GBC cell growth and proliferation.

\section{Downregulation of SLC25A22 suppressed metastasis of GBC in vitro via EMT}

To validate whether the metastasis was affected by SLC25A22 in GBC cells, a wound healing assay and transwell assay were conducted in GBC-SD and NOZ cells transfected with shSLC25A22/shNC. As shown in Fig. $4 a, b$, migration and invasion ability of the shRNA group was dramatically suppressed compared with control group. Furthermore, we examined the expression of EMT-related protein (E-cadherin, N-cadherin, Vimentin) in groups above mentioned using western-blot (Fig. 4c), which suggests that SLC25A22 may have an effect on metastasis of GBC cells through the EMT processes.

\section{Inhibition of SLC25A22 promoted mitochondrial-related apoptosis in GBC cells via MAPK/ERK pathway}

In light of the suppressive effect on GBC cell progression by downregulating SLC25A22, we separately carried out an apoptosis analysis for two groups of GBC-SD and NOZ cells (shSLC25A22/shNC) using Annexin V/PI Apoptosis Kit and flow cytometry (Fig. 5a). Our results indicated that downregulation of SLC25A22 led to a significant increase in the number 


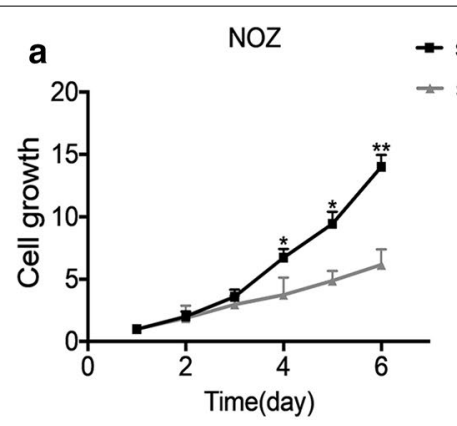

b
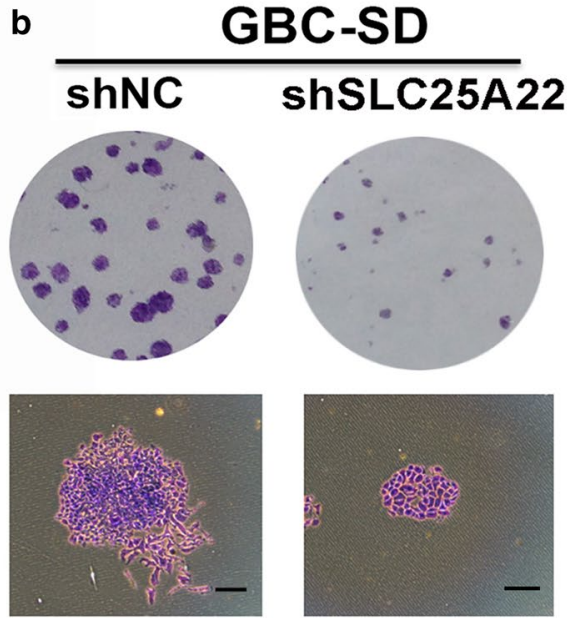

C

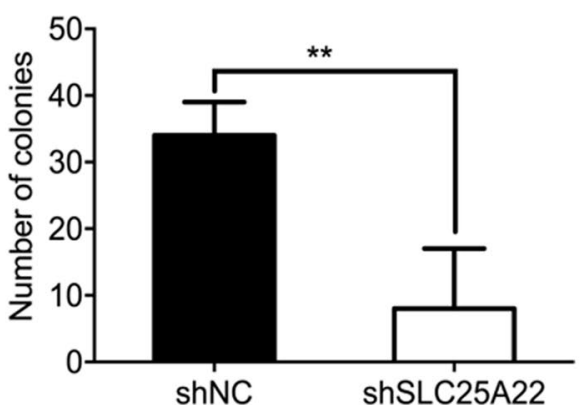

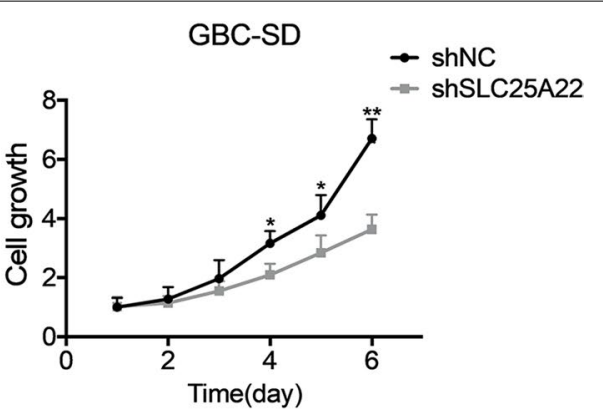

NOZ
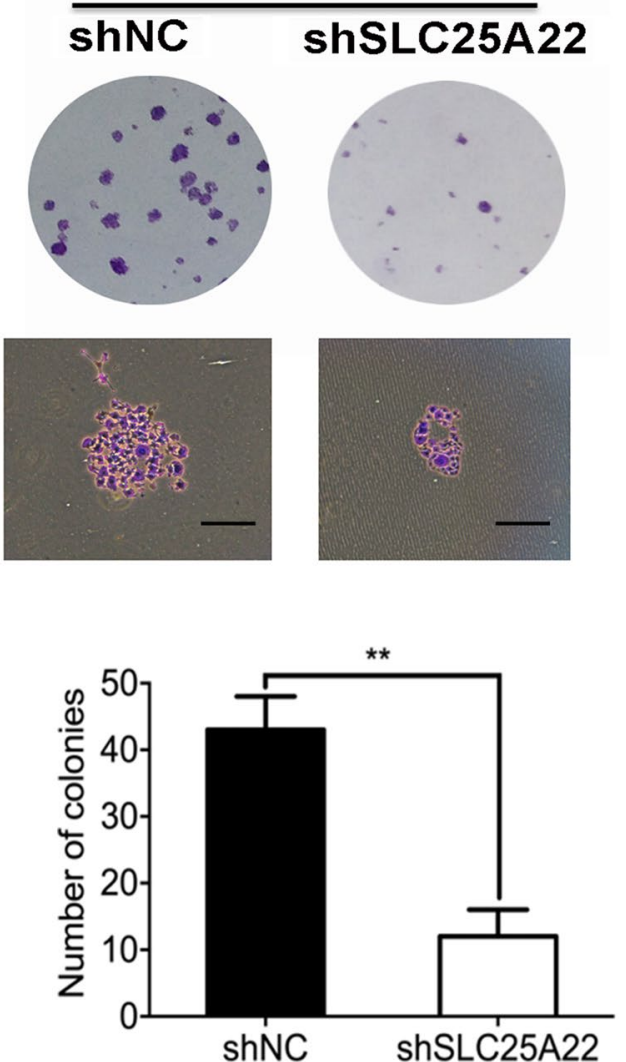

Fig. 2 Block of SLC25A22 inhibited growth in vivo. a The viability of GBC-SD and NOZ transfected with shSLC25A22 and shNC were analyzed using CCK8 viability assays $\left({ }^{*} p<0.05,{ }^{* *} p<0.01\right)$. b, c Cell colony formation displayed cell growth suppression when SLC25A22 was knocked down (shSLC25A22) compared to the control group (shNC). The number of colonies are shown in charts $\left({ }^{* *} p<0.01\right)$

of early apoptotics cells. To further explore the molecular mechanism for this alteration, the expression of several apoptosis markers were detected by westernblot (Fig. 5b). Cleaved-PARP, Cytochrome-c and BAX, three markers representing a state of apoptosis, were found to be significantly increased in the shRNA group. In contrast, BCL-2 was apparently reduced by knockdown of SLC25A22. It has been reported that the deregulation of MEK/ERK phosphorylation plays an antiapoptotic role in various cancers [12-17]. To determine whether the MAPK/ERK signaling pathway is associated with SLC25A22-induced apoptosis, we estimated the protein levels of MEK/ERK using westernblot and indicated that the expression of p-MEK/p-ERK was obviously decreased when SLC25A22 was downregulated (Fig. 5c). Therefore, MAPK/ERK pathway was 

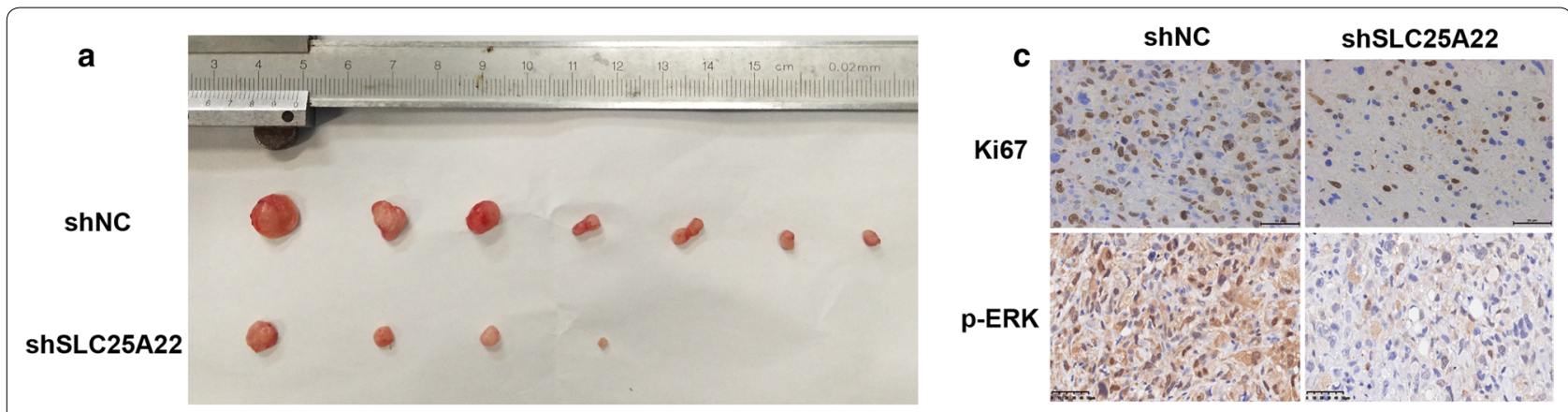

b

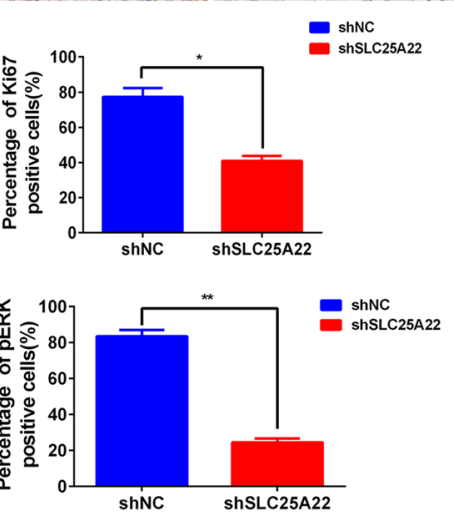

Fig. 3 SLC25A22 plays a vital role in GBC metastasis and proliferation in vivo. a SLC25A22 inhibited cell growth in vivo as observed with a subcutaneous xenograft model. $\mathbf{b}$ The tumor volumes were measured once a week from the 10th day and the results are shown on the line chart $\left({ }^{*} p<0.05,{ }^{* *} p<0.01\right)$. Tumor were weighed when the mice were sacrificed and the data was displayed on the scatter plot $\operatorname{chart}\left({ }^{*} p<0.05\right)$. $\mathbf{c}, \mathbf{d} \| \mathrm{HC}$ staining of tumor xenografts for Ki67 and p-ERK. The percentage of Ki67 and p-ERK positive cells are shown in the graph bar $\left({ }^{*} p<0.05,{ }^{* *} p<0.01\right)$

considered to play a vital role in apoptosis by knockdown of SLC25A22.

\section{Discussion}

Our results demonstrated there was high expression of SLC25A22 in gallbladder cancer and that it played a key role it played tumor progression including in vitro and in vivo. We focused on SLC25A22, a gene encodes mitochondrial glutamate transporter, that was shown by Wong et al. to promote proliferation and migration in colorectal cancer [10]. However, most research about SLC25A22 concentrated on its association neonatal epileptic encephalopathy [6-9], so the function of SLC25A22 and its molecular mechanism still remain unclear in malignant tumors. Interestingly, our microarray analysis demonstrated that there was an expression difference of SLC25A22 in GBC. The result of IHC staining and qRT-PCR analysis confirmed that SLC25A22 was a tumor-related protein.

As is well known, the aggressive proliferation capacity of metastasis is the most devastating feature of malignant tumor. Gallbladder cancer is a highly aggressive fatal neoplasm characterized by its strong invasiveness and rapid progression [18]. In the present study, the result of our experiments indicated that the metastasis and proliferation of the tumor were effectively inhibited by downregulating the expression of SLC25A22. The epithelial-to-mesenchymal transition (EMT) is a process related with tumor stemness, metastasis and resistance to therapy [19]. In our experiments, SLC25A22 was shown to be a significant factor in promoting tumor invasion and migration. E-cadherin, $\mathrm{N}$-cadherin and vimentin, are prominent markers of EMT [20]. Interestingly, our western-blot analysis demonstrated there was a close relationship between SLC25A22 and EMT. In other words, SLC25A22 might promote tumor cell invasion and metastasis via the EMT pathway in GBC.

The microenvironment of cancer cells and surrounding stromal cells with different genetic/epigenetic backgrounds is distinct from normal cells, which is called "intra-tumoral heterogeneity" [21]. The Warburg effect described as a tendency of most cancer cells to uptake glucose and convert it primarily to lactate in spite of available oxygen in cancer cells. Even though the Warburg effect has been widely recognized as an important feature of metabolic reprogramming, a lot of evidence has displayed that cancer cells also depend on mitochondrial metabolism $[22,23]$. It has been reported that the oncogene c-Myc, which is known to engage in glutamine catabolism that exceeds requirement for protein and 


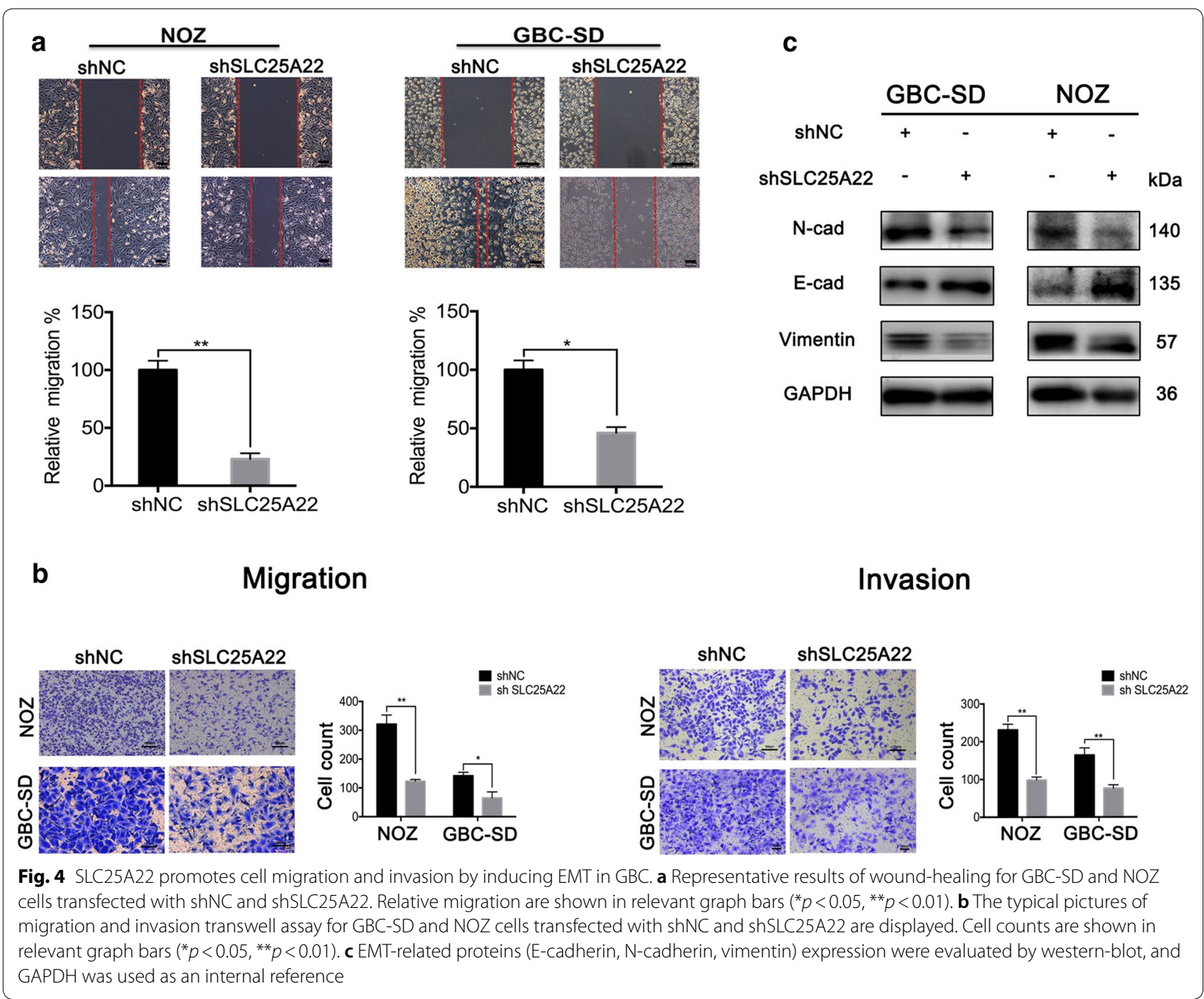

nucleotide biosynthesis by its transcriptional regulatory roles of repressing miR-23a and miR-23b [24]. As a result of c-Myc-mediated metabolic reprogramming, both glutamine uptake and glutamine catabolism are increased therefore the TCA cycle in mitochondria are frequently activated, which is called "glutamine addiction" [21, 25]. This is why the elevated expression and interaction of amino acid transporters, contributes to the activation of glutamine metabolic reprogramming and protects tumor cells against accumulation of oxidative stress mediated by cystine metabolic reprogramming. However, the complex mechanism of metabolic reprogramming due to c-Myc expression that regulates glutamine metabolism in cancer cells is still not well understood. Programmed cell death, also called apoptosis, was first defined by Lockshin \& Williams in the insect development [26]. Numerous studies have supported the conclusion that apoptosis plays a key role in regulating the development of malignant tumors. Mitochondrial outer membrane permeabilization (MMOP), a crucial process in intrinsic apoptotic pathway, was mediated by BCL-2-associated X protein (BAX) [27]. As a result, cytochrome-c was released from mitochondrial intermembrane to cytoplasm and induced to form apoptosome which indicated the occurrence of apoptosis [28]. In addition, cleaved PARP (poly-ADPribose polymerase) was highly considered to be a marker of activation in apoptosis $[29,30]$. Yet the MMOP process was inhibited by the anti-apoptotic member BCL-2. Due to the results of cell apoptosis analysis, SLC25A22 may promote tumor growth by suppressing the mitochondrial apoptosis in GBC. To further validate our opinion, we detected the proteins related with intrinsic apoptotic pathway as mentioned above by western-blot. Consistently, SLC25A22 promoted tumor progression by preventing the process of apoptosis via the mitochondrial pathway. 


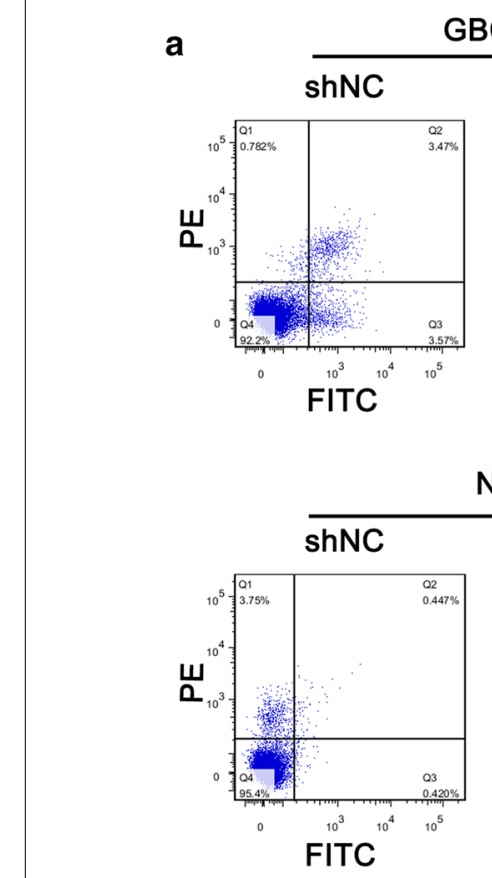

GBC-SD

NOZ

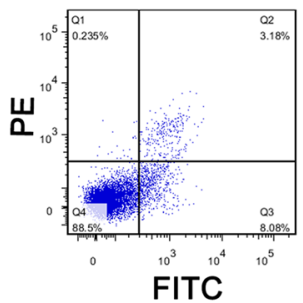

FITC
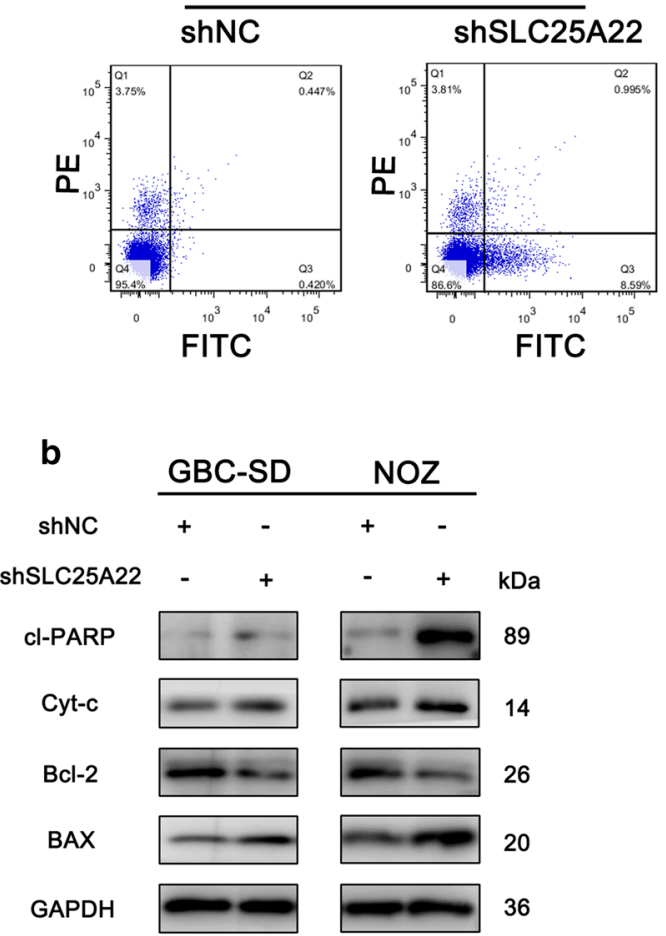

Fig. 5 SLC25A22 inhibits GBC cells apoptosis by regulating MAPK/ERK pathway. a Flow cytometry analysis of cell apoptosis of GBC-SD and NOZ cells with annexin V/PI staining indicating there was a significant difference of early apoptosis between two groups (shNC, shSLC25A22). $\mathbf{b}$ Apoptosis-related proteins (cleaved-PARP, Cytochrome-c, BCL-2, BAX) expression was analyzed by western-blot. c The protein of GBC-SD and NOZ cells were transfected with shNC and shSLC25A22 was subjected to analysis by western-blot. Representative immunoblots of MAPK/ERK pathway were analyzed with GAPDH as an internal reference
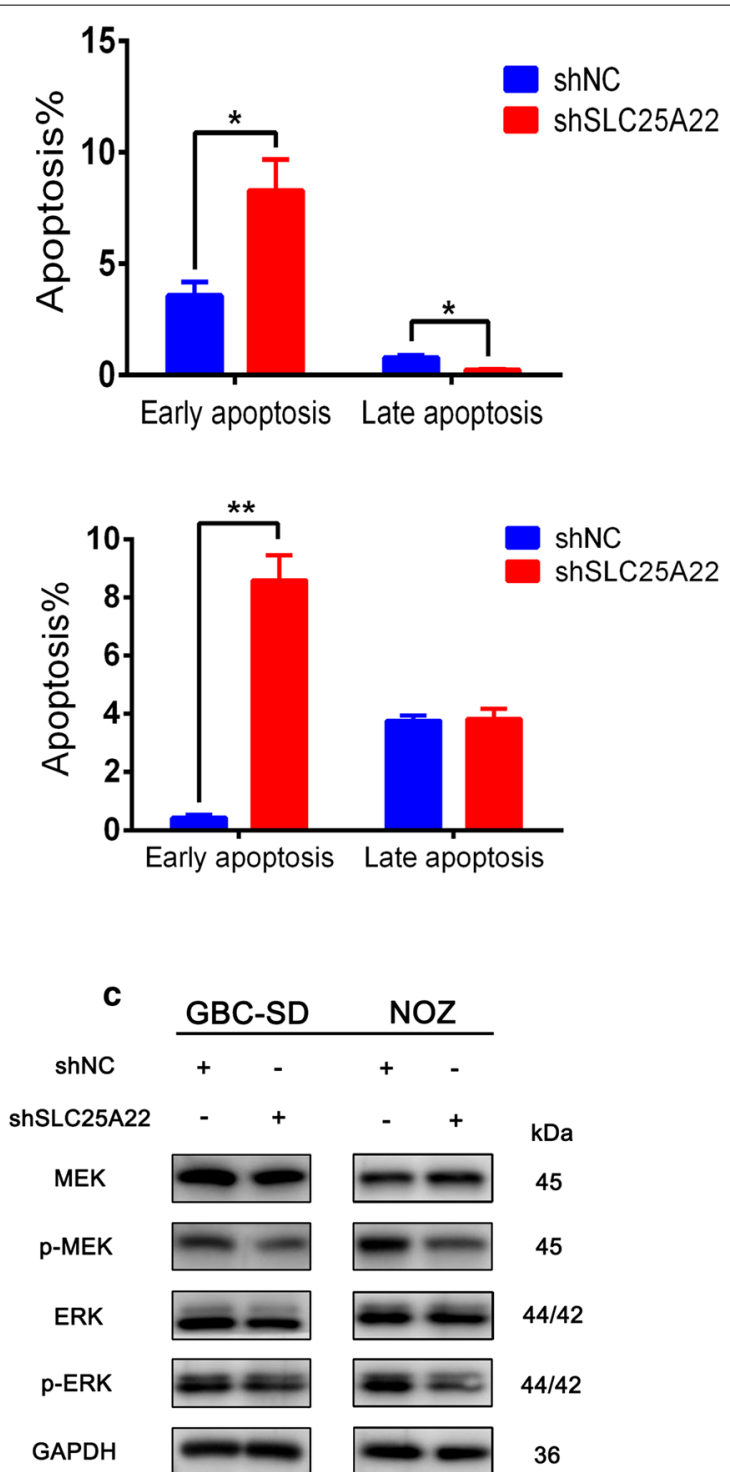

According to research by Wong et al., SLC25A22 knockdown caused the depletion of ATP as it activated AMPactivated protein kinase $\alpha(\mathrm{AMPK} \alpha)$ so that RAF/MEK/ ERK cascade was suppressed in CRC. Therefore, we focused on the MAPK/ERK pathway, a classical signaling pathway reported to regulate tumor development including proliferation, EMT, apoptosis, inflammation, and immunity [31-36]. The previous study has confirmed that MAPK/ ERK was involved in BAX-mediated apoptosis. In the present study, we further demonstrated that downregulation 
via altering mitochondria-associated apoptosis process mediated by MAPK/ERK pathway. Thus, SLC25A22 has a potential to be used as a target for cancer diagnosis of $\mathrm{GBC}$ and related therapies.

\begin{abstract}
Abbreviations
MCS: mitochondrial carrier system; GBC: gallbladder cancer; qRT-PCR: quantitative real-time PCR; IHC: immunohistochemistry; MAPK: mitogen-activated protein kinase; ERK: extracellular regulated protein kinases; pERK: phosphorylated extracellular regulated protein kinases; CRC: colorectal cancer; FBS: fetal bovine serum; CCK-8: cell counting kit-8; HRP: horse radish peroxidase; PRAP: poly ADP-ribose polymerase; BCL-2: B-cell lymphoma-2; BAX: B-cell lymphoma-2-associated X protein; GAPDH: glyceraldehyde-3-phosphate dehydrogenase; FITC: fluorescein isothiocyanate; PI: propidium iodide; PBS: phosphate buffer saline; EMT: epithelial-to-mesenchymal transition; MMOP: mitochondrial outer membrane permeabilization; AMPKa: AMP-activated protein kinase a.
\end{abstract}

\section{Authors' contributions}

PD and LF designed the study; PD HL and XF conducted the experiments; CW, PW collected tissue samples; HC, BZ, RZ purchased reagents; JZ, SH, BL collected and arranged the data. PD and LF wrote the main manuscript text. All authors read and approved the final manuscript.

\section{Author details}

${ }^{1}$ Department of General Surgery, Second Affiliated Hospital of Nanchang University, No. 1 Minde Road, Nanchang 330006, China. ${ }^{2}$ Jiangxi Province Key Laboratory of Molecular Medicine, No. 1 Minde Road, Nanchang 330006 China. ${ }^{3}$ Department of General Surgery, Xinhua Hospital Affiliated to Shanghai Jiao Tong University School of Medicine, No. 1665 Kongjiang Road, Shanghai 200092, China. ${ }^{4}$ Department of General Surgery, First Affiliated Hospital of Nanchang University, No. 17 Yongwai Main Street, Nanchang 330006, China.

\section{Acknowledgements}

We are grateful to Shanghai Key Laboratory of Biliary Tract Disease Research (Shanghai, China) for providing research cooperation platform support. This study was supported in part by grants from the Key Research Project of Jiangxi Province, China (No. 20171BBG70063), Education Department of Jiangxi Province (No. GJJ150260), the Natural Science Foundation of Nation, China (No. 81760438) and the Innovation Research Foundation of Graduate School of Nanchang University (No. CX2017204). We would like to thank MD. Fei Zhang and PhD. Yijian Zhang for their valuable input on the manuscript.

\section{Competing interests}

The authors declare that they have no competing interests.

\section{Availability of data and materials}

The data are included within the manuscript.

\section{Consent for publication}

All the authors agree to the publication clause.

\section{Ethics approval and consent to participate}

All operations as described above were approved the Institutional Animal Care and Use Committee of Xinhua Hospital, School of Medicine, Shanghai Jiao Tong University. All animal treatments were conducted in accordance with the National Institutes of Health Guide for the Care and Use of Laboratory Animals.

\section{Funding}

This study was supported in part by grants from the Key Research Project of Jiangxi Province, China (No. 20171 BBG70063), Education Department of Jiangxi Province (No. GJJ150260), the Natural Science Foundation of Nation, China (No. 81760438) and the Innovation Research Foundation of Graduate School of Nanchang University (No. CX2017204).

\section{Publisher's Note}

Springer Nature remains neutral with regard to jurisdictional claims in published maps and institutional affiliations.

Received: 30 November 2018 Accepted: 4 February 2019

Published online: 14 February 2019

\section{References}

1. Zhang F, Xiang $S$, Cao Y, Li M, Ma Q, Liang H, Li H, Ye Y, Zhang Y, Jiang L, et al. EIF3D promotes gallbladder cancer development by stabilizing GRK2 kinase and activating PI3 K-AKT signaling pathway. Cell Death Dis. 2017;8(6):e2868.

2. Song XL, Zhang YJ, Wang XF, Zhang WJ, Wang Z, Zhang F, Zhang YJ, Lu $J H$, Mei JW, Hu YP, et al. Casticin induces apoptosis and G0/G1 cell cycle arrest in gallbladder cancer cells. Cancer Cell Int. 2017;17:9.

3. Li M, Liu F, Zhang F, Zhou W, Jiang X, Yang Y, Qu K, Wang Y, Ma Q, Wang T, et al. Genomic ERBB2/ERBB3 mutations promote PD-L1-mediated immune escape in gallbladder cancer: a whole-exome sequencing analysis. Gut. 2018. https://doi.org/10.1136/gutjnl-2018-316039.

4. Taylor EB. Functional properties of the mitochondrial carrier system. Trends Cell Biol. 2017;27(9):633-44.

5. Goubert E, Mircheva Y, Lasorsa FM, Melon C, Profilo E, Sutera J, Becq H, Palmieri F, Palmieri L, Aniksztejn L, et al. Inhibition of the mitochondrial glutamate carrier SLC25A22 in astrocytes leads to intracellular glutamate accumulation. Front Cell Neurosci. 2017:11:149.

6. Gursoy S, Ercal D. Diagnostic approach to genetic causes of early-onset epileptic encephalopathy. J Child Neurol. 2016;31(4):523-32.

7. Molinari F, Kaminska A, Fiermonte G, Boddaert N, Raas-Rothschild A, Plouin P, Palmieri L, Brunelle F, Palmieri F, Dulac O, et al. Mutations in the mitochondrial glutamate carrier SLC25A22 in neonatal epileptic encephalopathy with suppression bursts. Clin Genet. 2009;76(2):188-94.

8. Poduri A, Heinzen EL, Chitsazzadeh V, Lasorsa FM, Elhosary PC, LaCoursiere CM, Martin E, Yuskaitis CJ, Hill RS, Atabay KD, et al. SLC25A22 is a novel gene for migrating partial seizures in infancy. Ann Neurol. 2013;74(6):873-82.

9. Reid ES, Williams H, Anderson G, Benatti M, Chong K, James C, Ocaka L, Gosgene, Hemingway C, Little D, et al. Mutations in SLC25A22: hyperprolinaemia, vacuolated fibroblasts and presentation with developmental delay. J Inherit Metab Dis. 2017;40(3):385-94.

10. Wong CC, Qian Y, Li X, Xu J, Kang W, Tong JH, To K-F, Jin Y, Li W, Chen H, et al. SLC25A22 promotes proliferation and survival of colorectal cancer cells with KRAS mutations and xenograft tumor progression in mice via intracellular synthesis of aspartate. Gastroenterology. 2016;151(5):945. e946-960.e946.

11. Zhang F, Liu B, Wang Z, Yu XJ, Ni QX, Yang WT, Mukaida N, Li YY. A novel regulatory mechanism of Pim-3 kinase stability and its involvement in pancreatic cancer progression. Mol Cancer Res MCR. 2013;11(12):1508-20.

12. Shu YJ, Bao RF, Jiang L, Wang Z, Wang XA, Zhang F, Liang HB, Li HF, Ye YY, Xiang SS, et al. MicroRNA-29c-5p suppresses gallbladder carcinoma progression by directly targeting CPEB4 and inhibiting the MAPK pathway. Cell Death Differ. 2017;24(3):445-57.

13. Wang H, Shi J, Luo Y, Liao Q, Niu Y, Zhang F, Shao Z, Ding Y, Zhao L. LIM and SH3 protein 1 induces TGFbeta-mediated epithelial-mesenchymal transition in human colorectal cancer by regulating S100A4 expression. Clin Cancer Res. 2014;20(22):5835-47.

14. Imada K, Shiota M, Kohashi K, Kuroiwa K, Song Y, Sugimoto M, Naito S, Oda Y. Mutual regulation between Raf/MEK/ERK signaling and Y-boxbinding protein-1 promotes prostate cancer progression. Clin Cancer Res. 2013;19(17):4638-50.

15. Zhang JX, Xu Y, Gao Y, Chen C, Zheng ZS, Yun M, Weng HW, Xie D, Ye S. Decreased expression of miR-939 contributes to chemoresistance and metastasis of gastric cancer via dysregulation of SLC34A2 and Raf/MEK/ ERK pathway. Mol Cancer. 2017;16(1):18.

16. Yang Z, Zhao Y, Yao Y, Li J, Wang W, Wu X. Equol induces mitochondriadependent apoptosis in human gastric cancer cells via the sustained activation of ERK1/2 pathway. Mol Cells. 2016;39(10):742-9. 
17. Ma Y, Wang L, Neitzel LR, Loganathan SN, Tang N, Qin L, Crispi EE, Guo Y, Knapp S, Beauchamp RD, et al. The MAPK pathway regulates intrinsic resistance to BET inhibitors in colorectal cancer. Clin Cancer Res. 2017;23(8):2027-37.

18. Li M, Zhang Z, Li X, Ye J, Wu X, Tan Z, Liu C, Shen B, Wang XA, Wu W, et al. Whole-exome and targeted gene sequencing of gallbladder carcinoma identifies recurrent mutations in the ErbB pathway. Nat Genet. 2014;46(8):872-6.

19. Pastushenko I, Brisebarre A, Sifrim A, Fioramonti M, Revenco T, Boumahdi S, Van Keymeulen A, Brown D, Moers V, Lemaire S, et al. Identification of the tumour transition states occurring during EMT. Nature. 2018;556(7702):463-8

20. Shenoy AK, Jin Y, Luo H, Tang M, Pampo C, Shao R, Siemann DW, Wu L, Heldermon CD, Law BK, et al. Epithelial-to-mesenchymal transition confers pericyte properties on cancer cells. J Clin Investig. 2016;126(11):4174-86.

21. Yoshida GJ. Metabolic reprogramming: the emerging concept and associated therapeutic strategies. J Exp Clin Cancer Res CR. 2015;34:111.

22. Wise DR, DeBerardinis RJ, Mancuso A, Sayed N, Zhang XY, Pfeiffer HK, Nissim I, Daikhin E, Yudkoff M, McMahon SB, et al. Myc regulates a transcriptional program that stimulates mitochondrial glutaminolysis and leads to glutamine addiction. Proc Natl Acad Sci USA. 2008;105(48):18782-7.

23. Yoshida GJ. Emerging roles of Myc in stem cell biology and novel tumor therapies. J Exp Clin Cancer Res CR. 2018;37(1):173.

24. Gao P, Tchernyshyov I, Chang TC, Lee YS, Kita K, Ochi T, Zeller KI, De Marzo AM, Van Eyk JE, Mendell JT, et al. c-Myc suppression of miR-23a/b enhances mitochondrial glutaminase expression and glutamine metabolism. Nature. 2009;458(7239):762-5.

25. Timmerman LA, Holton T, Yuneva M, Louie RJ, Padro M, Daemen A, Hu M, Chan DA, Ethier SP, van't Veer LJ, et al. Glutamine sensitivity analysis identifies the $\mathrm{xCT}$ antiporter as a common triple-negative breast tumor therapeutic target. Cancer Cell. 2013;24(4):450-65.

26. Nagata S. Apoptosis and clearance of apoptotic cells. Annu Rev Immunol. 2018;36:489-517.

27. Jiang $X$, Jiang $H$, Shen Z, Wang $X$. Activation of mitochondrial protease OMA1 by Bax and Bak promotes cytochrome $c$ release during apoptosis. Proc Natl Acad Sci USA. 2014;111(41):14782-7.
28. Martinez-Fabregas J, Diaz-Moreno I, Gonzalez-Arzola K, Janocha S, Navarro JA, Hervas M, Bernhardt R, Velazquez-Campoy A, DiazQuintana A, De la Rosa MA. Structural and functional analysis of novel human cytochrome $C$ targets in apoptosis. Mol Cell Proteom MCP. 2014;13(6):1439-56.

29. Siegel C, McCullough LD. NAD+ depletion or PAR polymer formation: which plays the role of executioner in ischaemic cell death? Acta Physiol. 2011;203(1):225-34

30. Panja S, Ghate NB, Mandal N. A microalga, Euglena tuba induces apoptosis and suppresses metastasis in human lung and breast carcinoma cells through ROS-mediated regulation of MAPKs. Cancer Cell Int. 2016;16:51.

31. Peluso I, Yarla NS, Ambra R, Pastore G, Perry G. MAPK signalling pathway in cancers: olive products as cancer preventive and therapeutic agents. Semin Cancer Biol. 2017. https://doi.org/10.1016/j.semca ncer.2017.09.002.

32. Liu F, Yang $X$, Geng M, Huang M. Targeting ERK, an Achilles' Heel of the MAPK pathway, in cancer therapy. Acta Pharm Sin B. 2018;8(4):552-62.

33. Gao J, Zhao Y, Lv Y, Chen Y, Wei B, Tian J, Yang Z, Kong F, Pang J, Liu J, et al. Mirk/Dyrk1B mediates G0/G1 to S phase cell cycle progression and cell survival involving MAPK/ERK signaling in human cancer cells. Cancer Cell Int. 2013;13(1):2.

34. Latrasse D, Jegu T, Li H, de Zelicourt A, Raynaud C, Legras S, Gust A, Samajova O, Veluchamy A, Rayapuram N, et al. MAPK-triggered chromatin reprogramming by histone deacetylase in plant innate immunity. Genome Biol. 2017;18(1):131.

35. Apps JR, Carreno G, Gonzalez-Meljem JM, Haston S, Guiho R, Cooper JE, Manshaei S, Jani N, Holsken A, Pettorini B, et al. Tumour compartment transcriptomics demonstrates the activation of inflammatory and odontogenic programmes in human adamantinomatous craniopharyngioma and identifies the MAPK/ERK pathway as a novel therapeutic target. Acta Neuropathol. 2018;135(5):757-77.

36. Mulholland DJ, Kobayashi N, Ruscetti M, Zhi A, Tran LM, Huang J, Gleave $\mathrm{M}, \mathrm{Wu}$ H. Pten loss and RAS/MAPK activation cooperate to promote EMT and metastasis initiated from prostate cancer stem/progenitor cells. Can Res. 2012;72(7):1878-89.
Ready to submit your research? Choose BMC and benefit from:

- fast, convenient online submission

- thorough peer review by experienced researchers in your field

- rapid publication on acceptance

- support for research data, including large and complex data types

- gold Open Access which fosters wider collaboration and increased citations

- maximum visibility for your research: over $100 \mathrm{M}$ website views per year

At BMC, research is always in progress.

Learn more biomedcentral.com/submissions 